\title{
CALCULATION OF INTENSITIES OF TORSIONAL-ROTATIONAL BANDS IN THE IR ABSORPTION SPECTRUM OF HYDROGEN PEROXIDE
}

\author{
A. V. Shashok, M. B. Shundalov, \\ and G. A. Pitsevich
}

UDC 539.192:530.145.7

\begin{abstract}
We present the calculated intensity distributions in torsional-rotational IR absorption bands of hydrogen peroxide. The torsional components of the band intensities have been calculated based on the appropriate matrix element computations. The contribution of the rotational components has been calculated using the 3j-symbols technique. The calculations have proved the reliability of available data on rotational constants, barrier heights of internal rotation, and locations of torsional-rotational levels of hydrogen peroxide.
\end{abstract}

Key words: hydrogen peroxide, torsional-rotational spectrum, intensity calculations, 3j-symbols.

Introduction. Hydrogen peroxide is the simplest non-rigid molecule and has served for a long time [1-5] and continues to serve [6-9] as the subject of spectral research and quantum-chemical calculations of the geometry and electronic structure [10-15]. On one hand, this is due to the comparative simplicity of the molecular structure; on the other, to the ability of the hydroxyls to rotate internally and form a complicated torsional-rotational spectrum. Research of the last decades has shown convincingly that very simple peroxides and their fragments that are present in trace quantities in the upper layers of the atmosphere have a destructive effect on the ozone layer of the earth [12, 16]. Therefore, the study of the spectral characteristics of these compounds is of great interest.

Energy states, frequencies of torsional and torsional-rotational transitions, and group theory approaches to establishing selection rules in IR and Raman spectra have been calculated several times [4, 5, 7, 8, 17-20]. Nevertheless, intensities of rotational components of torsional transitions of hydrogen peroxide in the far IR region have not been calculated. Because heights of torsional barriers, the position of torsional-rotational energy levels, and rotational constants of $\mathrm{H}_{2} \mathrm{O}_{2}$ are used as starting data to calculate absorption band intensities due to torsional-rotational transitions, the agreement of the calculated and experimental band intensities would provide additional confirmation that the calculated frequencies of torsional-rotational spectra that were calculated earlier are correct.

Herein the intensity distribution in the torsional-rotational IR absorption of hydrogen peroxide is calculated based on computation of matrix elements of the dipole moment components and use of the $3 j$-symbols technique.

Experimental. It is known [21] that the integral extinction coefficient, which is responsible for the intensity distribution in the IR absorption spectrum, is defined by the expression

$$
\int \varepsilon(v) d v=\frac{8 \pi^{3} N_{\mathrm{A}} \tilde{v}_{i j}}{\left(4 \pi \varepsilon_{0}\right) 3 h c Q} \exp \left(-\frac{E_{i}-E_{0}}{k T}\right)\left[1-\exp \left(-\frac{E_{j}-E_{i}}{k T}\right)\right] S_{i j},
$$

where the factors containing exponentials take into account the population of the lower state and the probability of forced emission (which is important for narrowly separated energy levels), respectively; $Q=\Sigma g_{w} \exp \left[-E_{w} / k T\right]$, the sum over states (distribution function); and $S_{i j}$, the line strength.

The line strength $S_{i j}$ of electric dipole transition $i \rightarrow j$ in coordinates of XYZ with the origin at the molecular center of mass and axes parallel to those of the laboratory system has the form

*To whom correspondence should be addressed.

Belorussian State University, 4 Nezavisimosti Ave., Minsk, 220030, Belarus; e-mail: pitsevich@bsu.by. Translated from Zhurnal Prikladnoi Spektroskopii, Vol. 75, No. 2, pp. 153-158, March-April, 2008. Original article submitted May 4, 2007. 


$$
S_{i j}=\sum_{\Psi, \Psi^{\prime \prime} \Psi_{F=X, Y, Z}} \sum_{\Psi^{\prime}}\left|\left\langle\Psi^{\prime}\left|\mu_{F}\right| \Psi^{\prime \prime}\right\rangle\right|^{2}=3 \sum_{\Psi, \Psi^{\prime \prime}}\left|\left\langle\Psi^{\prime}\left|\mu_{Z}\right| \Psi^{\prime \prime}\right\rangle\right|^{2},
$$

where $\mu_{F}$ is the dipole moment component along the $F$ axis. Wave functions $\Psi$ are written as products of electronic, vibrational, torsional, and rotational components:

$$
\Psi=\Psi_{\mathrm{el}} \Psi_{\text {vib }} \Psi_{\text {tor }}(\gamma) \Psi_{\text {rot }}(\theta, \phi, \chi)
$$

where $\gamma$ is the angle of internal rotation and $\theta, \phi$, and $\chi$ are Euler angles that determine the orientation of the fixed molecular $(m) x y z$ coordinate system relative to the laboratory fixed $(L) X Y Z$ system. Equation (1) takes into account the fact that the dipole moment components in the fixed molecular coordinate system contribute equally to the intensity so that the factor 3 is omitted from hereon.

Writing the function $\Psi$ as a product [Eq. (2)] enables the integral [Eq. (1)] to be examined in the fixed molecular coordinate system where (if the vibronic state is unchanged) the dipole moment of the molecule is a function of only the internal rotation angle and is independent of the overall molecular orientation relative to the laboratory coordinate system. Because the hydrogen peroxide molecule is a slightly asymmetric top (degree of asymmetry $\kappa=-0.99223$ ) $[2,8]$, the model of a symmetric top was used to describe the rotational motion. The geometric parameters and rotational constants of $\mathrm{H}_{2} \mathrm{O}_{2}$ were taken from the literature [2], where the high-resolution IR absorption spectrum of $\mathrm{H}_{2} \mathrm{O}_{2}$ in the gas phase was recorded. The calculated heights of potential barriers of internal rotation were in excellent agreement with calculated and experimental band frequencies of the torsional-rotational spectrum of the molecule.

The dipole moment vector components in the laboratory coordinate system $L$ are expressed as its components in the fixed molecular system $\mathrm{m}$ as follows:

$$
\mu_{L}^{(1, \sigma)}=\sum_{\sigma^{\prime}=-1}^{1}\left[D_{\sigma \sigma^{\prime}}^{(1)}(\theta, \phi, \chi)\right]^{T} \mu_{m}^{\left(1, \sigma^{\prime}\right)}
$$

where $D_{\sigma \sigma^{\prime}}^{(1)}(\theta, \phi, \chi)$ is the first-order rotation matrix and, instead of Descartes coordinates of vector $\mu$ in coordinate system $L$, their linear combinations are used. These reflect the symmetry properties in rotational space:

$$
\mu_{L}^{(1,+1)}=\frac{1}{\sqrt{2}}\left(\mu_{X}+i \mu_{Y}\right), \mu_{L}^{(1,-1)}=\frac{1}{\sqrt{2}}\left(\mu_{X}-i \mu_{Y}\right), \quad \mu_{L}^{(1,0)}=\mu_{Z}
$$

and analogous expressions for components of $\mu$ in coordinate system $m$.

Then, taking into account Eq. (1), Eq. (3) acquires the form

$$
\mu_{L}^{(1,0)}=\sum_{\sigma^{\prime}=-1}^{1}\left[D_{0 \sigma^{\prime}}^{(1)}(\theta, \phi, \chi)\right]^{\mathrm{T}} \mu_{m}^{\left(1, \sigma^{\prime}\right)}
$$

and the matrix elements in the expression for the line strength can be written as

$$
\begin{gathered}
\left\langle\Psi^{\prime}\left|\mu_{Z}\right| \Psi^{\prime \prime}\right\rangle=\left\langle\Psi_{\mathrm{el}}^{\prime} \Psi_{\mathrm{vib}}^{\prime} \Psi_{\text {tor }}^{\prime} \Psi_{\text {rot }}^{\prime}\left|\mu_{L}^{(0,1)}\right| \Psi_{\mathrm{el}}^{\prime \prime} \Psi_{\mathrm{vib}}^{\prime \prime} \Psi_{\text {tor }}^{\prime \prime} \Psi_{\text {rot }}^{\prime \prime}\right\rangle \\
=\sum_{\sigma^{\prime}=-1}^{1}=\left\langle\Psi_{\mathrm{el}}^{\prime} \Psi_{\mathrm{vib}}^{\prime} \Psi_{\text {tor }}^{\prime}\left|\mu_{m}^{\left(1, \sigma^{\prime}\right)}\right| \Psi_{\mathrm{el}}^{\prime \prime} \Psi_{\mathrm{vib}}^{\prime \prime} \Psi_{\text {tor }}^{\prime \prime}\right\rangle \times\left\langle\Psi_{\text {rot }}^{\prime}\left|D_{0 \sigma}^{(1)^{\mathrm{T}}}\right| \Psi_{\text {rot }}^{\prime \prime}\right\rangle .
\end{gathered}
$$

Because the vibronic state does not change for the studied transitions, the quantity below is introduced for further calculations

$$
\mu_{m, 0}^{\left(1, \sigma^{\prime}\right)}=\left\langle\Psi_{\mathrm{el}_{0}} \Psi_{\mathrm{vib}_{0}}\left|\mu_{m}^{\left(1, \sigma^{\prime}\right)}\right| \Psi_{\mathrm{el}_{0}} \Psi_{\mathrm{vib}_{0}}\right\rangle
$$


where $\Psi_{\mathrm{el}_{0}}$ and $\Psi_{\text {vib }_{0}}$ are wave functions of the ground electronic and vibrational states and $\mu_{m, 0}^{\left(1, \sigma^{\prime}\right)}$ is the permanent dipole moment of the molecule that is a function only of the internal rotation angle $\gamma$.

Equation (1) can be written as follows:

$$
S_{i j}=\sum_{\Psi,,^{\prime} \Psi^{\prime \prime}}\left|\sum_{\sigma^{\prime}=-1}^{1}\left\langle\Psi_{\text {tor }}^{\prime}\left|\mu_{m, 0}^{\left(1, \sigma^{\prime}\right)}\right| \Psi_{\text {tor }}^{\prime \prime}\right\rangle \times\left\langle\Psi_{\text {rot }}^{\prime}\left|D_{0 \sigma^{\prime}}^{(1)^{\mathrm{T}}}\right| \Psi_{\text {rot }}^{\prime \prime}\right\rangle\right|^{2} .
$$

The first factor in Eq. (4) defines the contribution of the torsional transition to the intensity of the spectral line. Because the $z$ axis of the coordinate system $m$ coincides with the top symmetry axis, i.e., is parallel to the $\mathrm{O}-\mathrm{O}$ bond, whereas the $x$ axis is situated in the plane that divides the dihedral angle of $\mathrm{H}-\mathrm{O}-\mathrm{O}-\mathrm{H}$ in half, the dipole moment components along the $y$ and $z$ axes revert to zero and the dependence of the dipole moment of the molecule on angle $\gamma$ to the null approximation is defined by the expression

$$
\mu_{m, 0}^{(1, \pm 1)}(\gamma)=\mu_{0} \cos \frac{\gamma}{2}, \mu_{0}=\text { const }
$$

Thus, the torsion factor acquires the form:

$$
\left\langle\Psi_{\text {tor }}^{\prime}\left|\mu_{m, 0}^{\left(1, \sigma^{\prime}\right)}\right| \Psi_{\text {tor }}^{\prime \prime}\right\rangle=\mu_{0}\left\langle\Psi_{\text {tor }}^{\prime}\left|\cos \frac{\gamma}{2}\right| \Psi_{\text {tor }}^{\prime \prime}\right\rangle
$$

where the torsion functions are real expansions in trigonometric Fourier series $[2,18]$. We used the two quantum numbers $v_{t}$ (analog of the vibrational quantum number) and $\tau$ to classify the torsion states. The quantum number $v_{t}$ takes on whole values, each of which corresponds with the four values $\tau=1,2,3$, and 4 . The selection rules allow transitions between states with any vt and impose limitations on the $\tau$ values: $\tau^{\prime}=1 \leftrightarrow \tau^{\prime \prime}=3$ and $\tau^{\prime}=2 \leftrightarrow \tau^{\prime \prime}=4$ $[8,17]$.

The calculation of the rotational integrals in Eq. (4) using angular momentum theory techniques can be reduced to a calculation of the corresponding $3 j$-symbols [22]. For this, the rotational functions of the symmetric top must be written in terms of a rotation matrix and it must be taken into account that only one component $\sigma^{\prime}$ of vector $\mu$ in the $m$ system, namely $k^{\prime}-k^{\prime \prime}=\sigma^{\prime}$, is important for the rotational transition $J^{\prime} k^{\prime} \leftrightarrow J^{\prime \prime} k^{\prime \prime}$. Then, the second factor in Eq. (4), according to the literature [22], will be

$$
\left(2 J^{\prime}+1\right)\left(2 J^{\prime \prime}+1\right)\left(\begin{array}{ccc}
J^{\prime} & 1 & J^{\prime \prime} \\
-k^{\prime} & \sigma^{\prime} & k^{\prime \prime}
\end{array}\right)^{2}
$$

Additional conditions that specify the type of $3 j$-symbols are the selection rules for quantum numbers $J$ and $k$. For selection rules $\Delta k= \pm 1$ and $\Delta J=0$, the band intensities accumulate as a result of summing the whole group of transitions with different $J$ values because the frequencies of these transitions are practically the same:

$$
\Delta J=0 \rightarrow \Delta E=B\left((k+\Delta k)^{2}-k^{2}\right) .
$$

For transitions corresponding to selection rules $\Delta k= \pm 1$ and $\Delta J= \pm 1$, the energy difference is defined by the value of quantum numbers $k$ and $J$ :

$$
\begin{gathered}
\Delta J=+1 \rightarrow \Delta E=2 A(J+1)+B\left((k+\Delta k)^{2}-k^{2}\right), \\
\Delta J=-1 \rightarrow \Delta E=2 A J+B\left((k+\Delta k)^{2}-k^{2}\right),
\end{gathered}
$$

as a result of which there is no accumulation and the intensity of these transitions is much lower. Intensities would accumulate analogously for the condition $\Delta J= \pm 1$ together with $\Delta k=0$. 


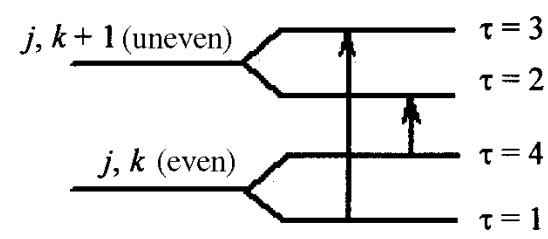

Fig. 1. Diagram of allowed torsional-rotational transitions for hydrogen peroxide.

TABLE 1. Wavenumbers $\left(v, \mathrm{~cm}^{-1}\right)$ and Relative Intensities $\left(I_{\text {rel }}\right)$ for Torsional Transitions in the IR Spectrum of Hydrogen Peroxide

\begin{tabular}{|c|c|c|c|}
\hline$v_{t}^{\prime} \rightarrow v_{t}^{\prime \prime}$ & $\tau^{\prime} \rightarrow \tau^{\prime \prime}$ & $v$ & $I_{\text {rel }}$ \\
\hline $0 \rightarrow 0$ & $1 \rightarrow 3,2 \rightarrow 4$ & 11.25 & 0.217 \\
$0 \rightarrow 1$ & $3 \rightarrow 1,4 \rightarrow 2$ & 242.17 & 1.000 \\
$0 \rightarrow 1$ & $1 \rightarrow 3,2 \rightarrow 4$ & 369.07 & 0.535 \\
$1 \rightarrow 1$ & $1 \rightarrow 3,2 \rightarrow 4$ & 115.65 & 0.585 \\
$1 \rightarrow 2$ & $3 \rightarrow 1,4 \rightarrow 2$ & 197.45 & 0.591 \\
$2 \rightarrow 2$ & $1 \rightarrow 3,2 \rightarrow 4$ & 205.19 & 0.295 \\
\hline
\end{tabular}

Thus, the expressions for the $3 j$-symbols (for summation over $J$ ) are

$$
\sum_{J=k^{\prime}>k^{\prime \prime}}^{\infty}\left(\begin{array}{ccc}
J & 1 & J \\
-k^{\prime} & -1 & k^{\prime \prime}
\end{array}\right) \text { or } \sum_{J=k^{\prime \prime}>k^{\prime}}^{\infty}\left(\begin{array}{ccc}
J & 1 & J \\
-k^{\prime} & 1 & k^{\prime \prime}
\end{array}\right) \text {. }
$$

Next, the parity coupling factor of rotational quantum number $k$ and torsional $\tau$ [8], according to which even values of $k$ correspond to $\tau=1$ and 4 ; uneven, $\tau=2$ and 3, must be considered. All these limitations result in the absorption spectrum exhibiting two band progressions in $k$ with a constant frequency difference for identical $k$ values that is equal to twice the distance between torsional energy levels (Fig. 1).

Discussion. Table 1 gives the calculated intensities of torsional transitions taking into account matrix elements (5).

Figure 2 shows the calculated torsional (without taking into account rotational components) absorption spectrum of $\mathrm{H}_{2} \mathrm{O}_{2}$.

Intensities of torsional-rotational bands were calculated using all aforementioned factors. Table 2 gives the calculated and experimental wavenumbers and the corresponding transmittance for the main torsional-rotational transitions that form the absorption bands in the IR spectrum of $\mathrm{H}_{2} \mathrm{O}_{2}$ at $30-350 \mathrm{~cm}^{-1}$ with an indication of the initial and final quantum numbers that characterize the torsional-rotational state.

The torsional-rotational transitions between states with $v_{t}^{\prime}>0$ and $v_{t}^{\prime \prime}>1$ make a smaller contribution to the band intensity. However, they are taken into account in constructing the torsional-rotational absorption spectrum (Fig. 3).

In general, the calculations adequately reproduce trends in the intensity distribution of the torsional-rotational bands in the IR absorption spectrum of $\mathrm{H}_{2} \mathrm{O}_{2}$. Some of the differences in the calculated and experimental results can be explained by the following factors.

1) Use of the model of a rigid symmetric top as the initial approximation (because the inertial parameters of the molecule are weak functions of the rotational quantum numbers, the wavenumbers of the maxima of each individual envelope of an absorption band differ little and, therefore, the calculated intensity should correlate well with the integrated intensity of the bands in the IR absorption spectrum);

2) The presence in the spectrum of bands for the $P$ - and $R$-branches that form a continuous background that contributes relatively unevenly to the observed band intensity, distorting the true intensity of weak absorption bands; 


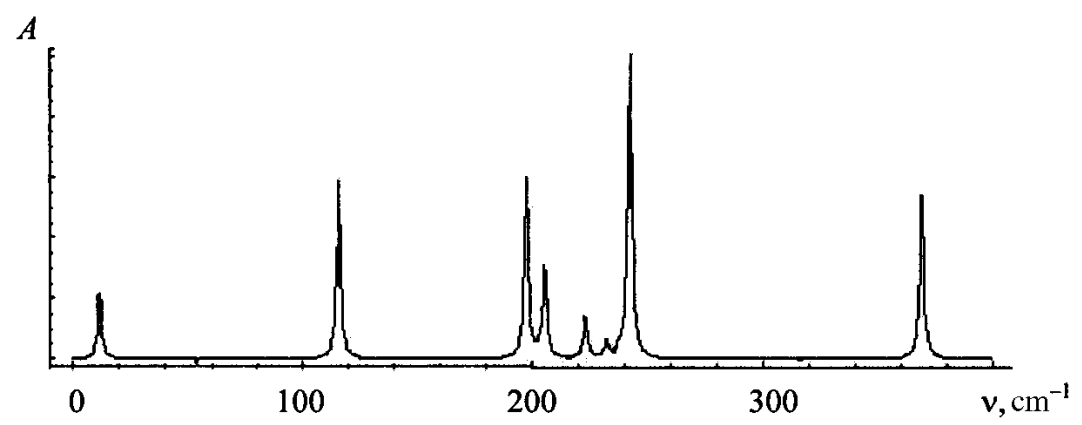

Fig. 2. Model torsional absorption spectrum of hydrogen peroxide.

TABLE 2. Wavenumbers $\left(v, \mathrm{~cm}^{-1}\right)$ and Transmittance $(T, \%)$ for Torsional-Rotational Bands in the IR Absorption Spectrum of Hydrogen Peroxide

\begin{tabular}{|c|c|c|c|c|c|}
\hline \multirow{2}{*}{$v_{t}^{\prime} \tau^{\prime} \rightarrow v_{t}^{\prime \prime} \tau^{\prime \prime}$} & \multirow{2}{*}{$k^{\prime} \rightarrow k^{\prime \prime}$} & \multicolumn{2}{|c|}{ Calculated } & \multicolumn{2}{|c|}{ Experimental [2] } \\
\hline & & $v$ & $T$ & $v$ & $T$ \\
\hline \multirow{7}{*}{$\begin{array}{l}01 \rightarrow 03 \\
02 \rightarrow 04\end{array}$} & $0 \rightarrow 1$ & 38.86 & 52 & - & - \\
\hline & $1 \rightarrow 2$ & 57.27 & 61 & 57.38 & 60 \\
\hline & $2 \rightarrow 3$ & 75.68 & 62 & 75.67 & 50 \\
\hline & $3 \rightarrow 4$ & 94.10 & 56 & 94.08 & 45 \\
\hline & $4 \rightarrow 5$ & 112.53 & 46 & 112.27 & 42 \\
\hline & $5 \rightarrow 6$ & 130.96 & 34 & 130.33 & 40 \\
\hline & $6 \rightarrow 7$ & 149.40 & 22 & 148.37 & 26 \\
\hline \multirow{8}{*}{$\begin{array}{l}03 \rightarrow 01 \\
04 \rightarrow 02\end{array}$} & $0 \rightarrow 1$ & 16.32 & 26 & - & - \\
\hline & $1 \rightarrow 2$ & 34.70 & 42 & 34.56 & 45 \\
\hline & $2 \rightarrow 3$ & 53.08 & 48 & 52.94 & 55 \\
\hline & $3 \rightarrow 4$ & 71.45 & 46 & 71.36 & 62 \\
\hline & $4 \rightarrow 5$ & 89.82 & 39 & 89.69 & 55 \\
\hline & $5 \rightarrow 6$ & 108.18 & 30 & 107.90 & 38 \\
\hline & $6 \rightarrow 7$ & 126.53 & 21 & 126.76 & 30 \\
\hline & $7 \rightarrow 8$ & 144.88 & 14 & 144.55 & 28 \\
\hline \multirow{16}{*}{$\begin{array}{l}03 \rightarrow 11 \\
04 \rightarrow 12\end{array}$} & $8 \rightarrow 9$ & 163.22 & 10 & 161.98 & 43 \\
\hline & $-7 \rightarrow-6$ & 140.87 & 15 & 142.19 & 20 \\
\hline & $-6 \rightarrow-5$ & 159.30 & 24 & 160.71 & 31 \\
\hline & $-5 \rightarrow-4$ & 177.72 & 35 & 179.14 & 38 \\
\hline & $-4 \rightarrow-3$ & 196.13 & 47 & 196.75 & 35 \\
\hline & $-3 \rightarrow-2$ & 214.54 & 56 & 215.74 & 45 \\
\hline & $-2 \rightarrow-1$ & 232.94 & 63 & 234.20 & 50 \\
\hline & $-1 \rightarrow-0$ & 251.33 & 66 & 251.34 & 50 \\
\hline & $0 \rightarrow 1$ & 269.72 & 64 & 269.42 & 47 \\
\hline & $1 \rightarrow 2$ & 288.10 & 59 & 288.42 & 43 \\
\hline & $2 \rightarrow 3$ & 306.48 & 50 & 307.10 & 32 \\
\hline & $3 \rightarrow 4$ & 324.85 & 39 & 324.46 & 52 \\
\hline & $4 \rightarrow 5$ & 343.22 & 28 & 343.41 & 25 \\
\hline & $5 \rightarrow 6$ & 361.58 & 18 & 363.94 & 27 \\
\hline & $6 \rightarrow 7$ & 379.93 & 12 & 279.44 & 27 \\
\hline & $7 \rightarrow 8$ & 398.28 & 8 & 399.01 & 20 \\
\hline
\end{tabular}




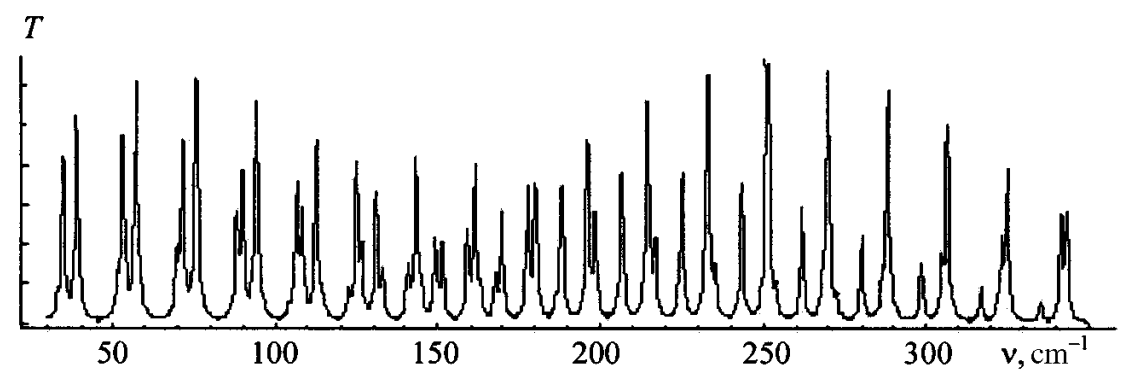

Fig. 3. Model torsional-rotational spectrum of hydrogen peroxide.

3) The superposition of spectral envelopes of closely spaced lines (because the envelopes were not separated during the experimental measurement, the line intensity at the maximum is distorted).

Conclusion. It was shown that the relative intensities of absorption bands of $\mathrm{H}_{2} \mathrm{O}_{2}$ in the far IR region can be calculated using a weak coupling of the vibrational, torsional, and rotational motions. The contribution to the absorption band intensities due to a change of the rotational state of the molecule can be estimated using a calculation of $3 j$-symbols. This reduces the calculation of series integrals to algebraic operations. The calculations confirm that the employed model parameters (equilibrium geometry of the molecule and internal rotational potential energy function) and the energies of fixed states of torsional and rotational motions of $\mathrm{H}_{2} \mathrm{O}_{2}$ are adequate.

\section{REFERENCES}

1. R. L. Redington, W. B. Olson, and P. C. Cross, J. Chem. Phys., 36, 1311-1326 (1962).

2. R. H. Hunt, R. A. Leacock, C. W. Peters, and K. T. Hecht, J. Chem. Phys., 42, 1931-1942 (1965).

3. R. H. Hunt and R. A. Leacock, J. Chem. Phys., 45, 3141-3147 (1966).

4. P. Helminger, W. C. Bowman, and F. C. DeLucia, J. Mol. Spectrosc., 85, 120-130 (1981).

5. W. C. Bowman, F. C. DeLucia, and P. Helminger, J. Mol. Spectrosc., 87, 575-577 (1981).

6. J. J. Hillman, D. E. Jennings, W. B. Olson, and A. Goldman, J. Mol. Spectrosc., 117, 46-59 (1986).

7. W. B. Olson, R. H. Hunt, B. W. Young, A. G. Maki, and J. W. Braultet, J. Mol. Spectrosc., 127, 12-34 (1988).

8. J.-M. Flaud, C. Camy-Peyret, J. W. C. Johns, and B. Carli, J. Chem. Phys., 91, 1504-1510 (1989).

9. C. Camy-Peyret, J.-M. Flaud, J. W. C. Johns, and M. Noel, J. Mol. Spectrosc., 155, 84-104 (1992).

10. P. A .Giguere and T. K. K. Srinivasan, J. Mol. Spectrosc., 66, 168-170 (1977).

11. D. Cremer and D. Christen, J. Mol. Spectrosc., 74, 480-482 (1979).

12. J. Koput, J. Mol. Spectrosc., 115, 438-441 (1986).

13. A. Chung-Phillips and K. A. Jebber, J. Chem. Phys., 102, 7080-7087 (1995).

14. J. Koput, Chem. Phys. Lett., 236, 516-520 (1995).

15. D. Du, A. Fu, and Z. Zhou, J. Mol. Struct. (THEOCHEM), 717, 127-132 (2005).

16. L. T. Molina and M. J. Molina, J. Phys. Chem., 91, 433-436 (1987).

17. J. T. Hougen, Can. J. Phys., 62, 1392-1402 (1984).

18. M. B. Shundalov and G. A. Pitsevich, Zh. Prikl. Spektrosk., 67, No. 4, 450-452 (2000).

19. M. B. Shundalov and G. A. Pitsevich, Zh. Prikl. Spektrosk., 68, No. 6, 699-703 (2001).

20. S. Carter and N. C. Handy, Spectrochim. Acta A, 60, 2107-2111 (2004).

21. P. R. Bunker and P. Jensen, Molecular Symmetry and Spectroscopy, NRC Research Press, Ottawa (1998).

22. R. N. Zare, Angular Momentum: Understanding Spatial Aspects in Chemistry and Physics, Wiley, New York (1988). 